\title{
Can Age Adjusted Charlson Comorbidity Index Predict Prognosis in Hospitalised COVID-19 patients?
}

\author{
Khurram Shahzad Khan* ${ }^{(D}$, Hisham El-Zanati, and Sajid Mahmud \\ ${ }^{1}$ Department of Surgery, University Hospital Hairmyres, East Kilbride, Scotland, UK
}

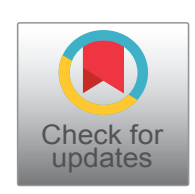

\begin{abstract}
Background: Age adjusted Charlson comorbidity index (ACCI) has previously shown to predict outcome in wide range of diseases. We aim to assess if ACCI can predict 30-day all-cause mortality and need for critical care in COVID-19 patients.

Methods: Prospective cohort study of hospitalised patients with confirmed COVID-19 in three acute hospitals. Patients were divided into two groups based on ACCl: Low ACCI (0-4) and high ACCI ( $\geq 5)$. Electronic case notes were analysed and results were compared.

Results: 173 patients were identified, 108 (62.4\%) were males, mean age was $68.4 \pm 14.6$ years. Median ACCI was 4 (range 0-11). 108 (62.4\%) patients were in low ACCI. High ACCI had more $\geq 71$ years-old patients (30.6\% vs. 81.5\%, p< $0.001)$. Low $\mathrm{ACCl}$ had more patients with normal renal function $(78.7 \%$ vs. $44.6 \%, p<0.001)$ and more likely to present with COVID-19 symptoms ( $87.0 \%$ vs. $56.9 \%, \mathrm{p}<0.001)$. More patients in low ACCI were transferred to critical care $(42.6 \%$ vs. $3.1 \%, p<0.001)$ and intubated $(30.6 \%$ vs. $1.5 \%, p<0.001)$. However, 30 -day all-cause mortality and overall poor outcome was similar in both groups, ( $15.7 \%$ vs. $23.1 \%, p=0.229$ and $35.2 \%$ vs. $24.6 \%, p=0.146$, respectively).
\end{abstract}

Conclusion: The outcome in COVID-19 hospitalised patients cannot be reliably predicted by ACCI.

Keywords

Covid-19, Pandemic, Outcome, Age Adjusted Charlson Comorbidity Index, 30-Day Mortality

\section{Abbreviations}

COPD: Chronic obstructive pulmonary disease (COPD); CVD: Cerebrovascular disease; ACCI: Age adjusted Charlson comorbidity index; PVD: Peripheral vascular disease; RT-PCR: Reverse transcriptase polymerase chain reaction; CXR: Chest X-ray; BSTI: British Society of Thoracic Imaging; DNAR: Do Not Attempt Resuscitation; LOS: Length of stay

\section{Introduction}

Since the diagnosis of the first case of COVID-19 in China in late December 2019 the disease has been spreading quite rapidly to the rest of the world resulting in a pandemic with a relatively high mortality compared to other respiratory viruses. It has been noticed throughout the world that patients with other underlying co-morbidities and elderly patients have a particularly poor prognosis with high morbidity and mortality from the disease with several studies from China and Italy reporting this. This included mainly hypertension, cardiac disease, chronic obstructive pulmonary disease (COPD), cerebrovascular disease (CVD) and diabetes mellitus [1-3].

One of the most popular comorbidity indices is the Charlson Comorbidity index $(\mathrm{CCl})$. The $\mathrm{CCl}$ was developed in the late 1980 s based on the 1-year mortality data from internal medicine patients admitted to a single New York Hospital and was also shown to predict 10 year mortality on long term follow up [4]. This index was later validated in a cohort of pa- tients with breast cancer [5]. It includes 19 medical conditions weighted 1-6 with total scores ranging from 0 to 37: Myocardial infarction, congestive heart failure, peripheral vascular disease (PVD), CVD, dementia, COPD, connective tissue disease, peptic ulcer disease, mild liver disease, diabetes mellitus, hemiplegia, moderate-severe renal disease, any tumour within the last 5 years, lymphoma, leukemia, moderate-severe liver disease, metastatic solid tumour, and AIDS [6]. The

*Corresponding author: Khurram Shahzad Khan, Surgical Registrar, Department of General Surgery, University Hospital Hairmyres, East Kilbride, G75 8RG, Scotland, UK, Tell: 0044 7533537292

Accepted: November 28, 2020

Published online: November 30, 2020

Citation: Khan KS, El-Zanati H, Mahmud S (2020) Can Age Adjusted Charlson Comorbidity Index Predict Prognosis in Hospitalised COVID-19 patients?. Ann Public Health Reports 4(1):115121 
Citation: Khan KS, El-Zanati H, Mahmud S (2020) Can Age Adjusted Charlson Comorbidity Index Predict Prognosis in Hospitalised COVID-19 patients?. Ann Public Health Reports 4(1):115-121

age adjusted $\mathrm{CCl}(\mathrm{ACCl})$ was later on introduced and is based on the same weighted co morbid medical conditions but includes a factor for age by decade: Beyond 50 years of age, 1 point is added for each decade. This combined age-comorbidity score was validated by Charlson [7] in 1994.

The $\mathrm{ACCl}$ has been shown to predict outcomes and survival in multiple previous studies in relation to a wide spectrum of various other conditions and in hospitalised patients in general. This included acute myocardial infarction, acute mesenteric ischemia, systemic lupus erythematosus, cerebrovascular stroke, renal disease, critical care patients and patients seen in the emergency department with sepsis [8-15]. Given the above and what we have now come to learn about the coronavirus it is possible that the usefulness of $\mathrm{ACCl}$ in predicting outcomes can be extended to patients with COVID-19 patients which would be very useful in planning resources and formulating management plans including difficult decisions on the ceiling of care of these patients and facilitating having realistic discussions with the patients' family members. This is particularly important given the limited critical care capacity in most hospitals which has been a major issue in most parts of the world in dealing with this pandemic.

The primary aim of our study was to assess if $\mathrm{ACCl}$ can predict 30-day all-cause mortality in COVID-19 positive hospitalised patients. The secondary aim was to analyse if $\mathrm{ACCl}$ can predict the need for critical care, total hospital stay and time to death.

\section{Materials and Methods}

This was a prospective observational study carried out on all hospitalized patients with reverse transcriptase polymerase chain reaction (RT-PCR) confirmed COVID-19, on Friday the $9^{\text {th }}$ April 2020. Patients from three acute hospitals in a single National Health Service (NHS) Trust in Scotland serving a population of 655,000 with over 1,660 in-patient's bed capacity were included. COVID-19 positive patients were identified from Trak Care Electronic Medical Record System. Patients with first negative swab that went to have further positive swabs were also included in the study. The electronic case notes were analysed for baseline characteristics and admission investigations.

The reason for admission was divided into three group: Group 1 was patients admitted with suspicion of COVID-19 infection, group 2 was patients presented with other pathologies but on admission (or within 7 days of admission) were found to have COVID-19 infection and group 3 was patients who were admitted with other pathologies and contracted COVID-19 infection in hospital (positive RT-PCR after 7 days of admission). For the patients in group 2 and 3 , the investigations were taken from the date of positive RT-PCR.

Scottish Index of Multiple Deprivation [16] were also calculated using the residential address. Index chest X-ray (CXR) was reported by consultant radiologist using British Society of Thoracic Imaging (BSTI) classification [17] who was blinded to the COVID-19 status.

Critical care was defined as care given above general ward level. Mortality was calculated for 30-day all-cause mortality. Poor outcome was defined as either need for intubation and/ or death. This was to account for those patients who were critically ill but not suitable for intubation and were Do Not Attempt Resuscitation (DNAR) and palliated. All patients were followed up for at least 30 days from admission or until discharge or death. Patients transferred to off-site rehabilitation unit were considered to be discharged.

Patients were divided into two groups based on $\mathrm{ACCl}$; Low ACCl (0-4) and high ACCI ( $\geq 5)$. The result were compared for baseline characteristics, blood results on admission and the outcome. For the length of stay (LOS) analysis, patients having ongoing inpatient care and who's who contracted the COVID-19 infection in hospital (group 3) were excluded.

This study was registered with the NHS Lanarkshire's Clin-

Table 1: Baseline characteristics.

\begin{tabular}{|c|c|c|c|}
\hline Sex & & Age & \\
\hline Male & $108(62.4 \%)$ & $<50$-years & $17(9.8 \%)$ \\
\hline \multirow[t]{2}{*}{ Female } & 65 (37.6\%) & $50-59$-years & $31(17.9 \%)$ \\
\hline & & $60-69$-years & $37(21.4 \%)$ \\
\hline Co morbidities & & $70-79$-years & $40(23.1 \%)$ \\
\hline Myocardial infarction & $20(11.6 \%)$ & $\geq 80$-years & $48(27.8 \%)$ \\
\hline Congestive cardiac failure & $7(4.0 \%)$ & & \\
\hline $\begin{array}{l}\text { Cerebrovascular accident or } \\
\text { transient ischemic attack }\end{array}$ & 30 (17.3\%) & Ethnicity & \\
\hline Hypertension & $88(50.9 \%)$ & White Scottish & $123(71.1 \%)$ \\
\hline $\begin{array}{l}\text { Chronic obstructive pulmonary } \\
\text { disease }\end{array}$ & 19 (11.0\%) & Other white ethnic group & $6(3.5 \%)$ \\
\hline Asthma & $20(11.6 \%)$ & Other African & $1(0.6 \%)$ \\
\hline Chronic kidney disease & 25 (14.5\%) & Pakistani & $1(0.6 \%)$ \\
\hline Diabetes Mellitus & $41(23.7 \%)$ & Not Recorded & $42(24.2 \%)$ \\
\hline Dementia & $26(15.0 \%)$ & & \\
\hline
\end{tabular}


Citation: Khan KS, El-Zanati H, Mahmud S (2020) Can Age Adjusted Charlson Comorbidity Index Predict Prognosis in Hospitalised COVID-19 patients?. Ann Public Health Reports 4(1):115-121

ical Quality Project, project id: 13134.

\section{Statistical analysis}

Qualitative data were given as frequency and percentages while quantitative data was expressed as the mean \pm S.D. Pearson uncorrected Chi-Square test was calculated in Stat pages to calculate $p$ value and odds ratio with $95 \%$ confidence interval. Student t-test was used to analyse length of stay (LOS) data. $P$ value of $<0.05$ was considered to be statistically significant.

\section{Results}

A total of 173 patients were identified. No patients were excluded from the study. Table 1 shows the baseline characteristics. $108(62.4 \%)$ of the patients were males, mean age was $68.4 \pm 14.6 y e a r s$. The most common comorbidity was hypertension (50.9\%). Figure 1 shows the distribution of $\mathrm{ACCl}$ in the cohort. The median ACCI was 4 (range 0 - 11). 108 (62.4\%) of patients were in the low $\mathrm{ACCl}$ group and 65 (37.6\%) of patients were in high $\mathrm{ACCl}$ group.

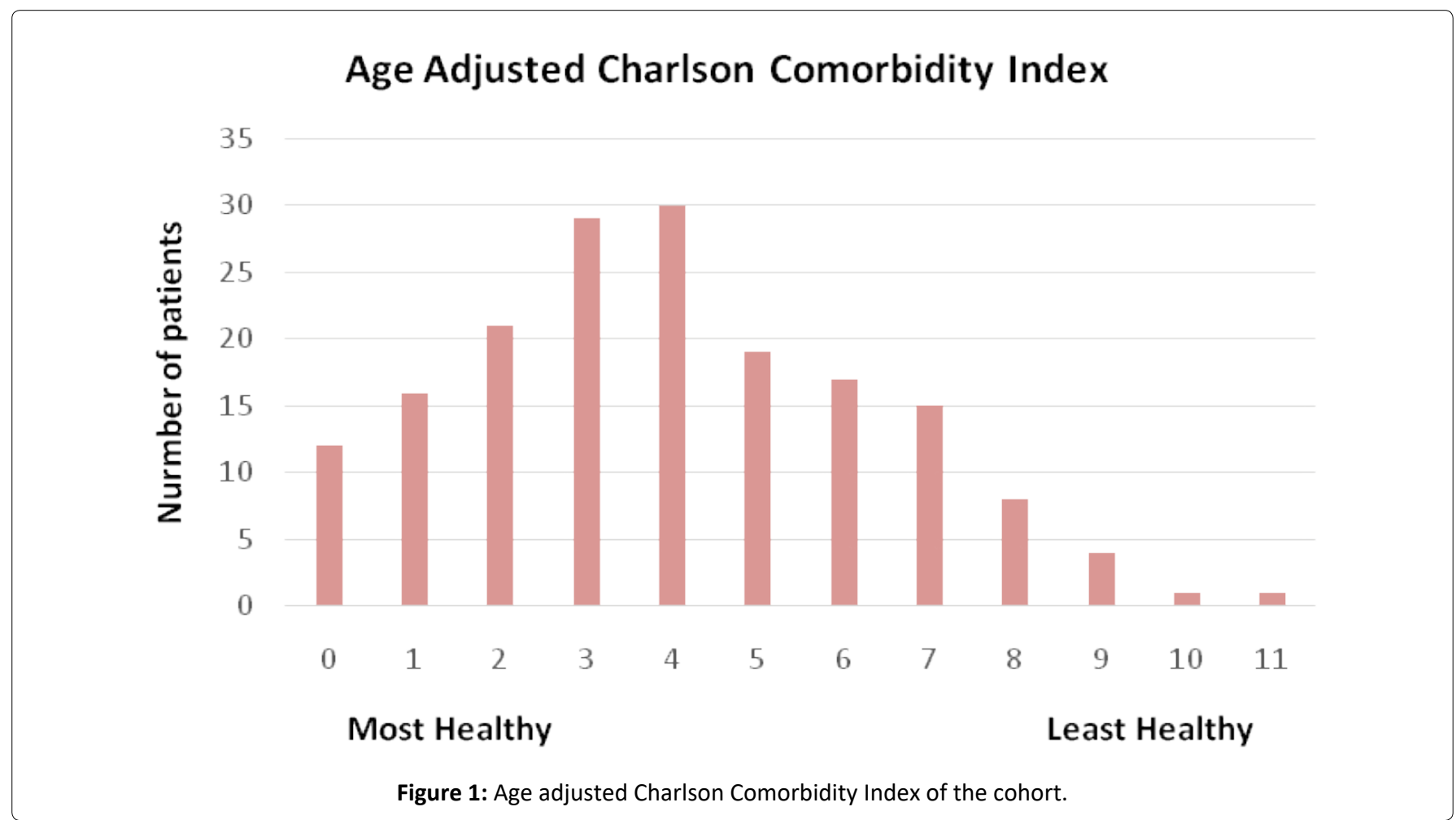

Table 2: Comparison of baseline characteristics, blood profile and reason of admission.

\begin{tabular}{|c|c|c|c|c|}
\hline & $\begin{array}{l}\text { Low ACCI } \\
\text { (n 108) }\end{array}$ & $\begin{array}{l}\text { High ACCI } \\
\text { (n 65) }\end{array}$ & P value & Odds Ratio (95\% Cl) \\
\hline & Low ACCI & $<50$-years & $17(9.8 \%)$ & \\
\hline (n 108) & High ACCI & 50 - 59-years & 31 (17.9\%) & \\
\hline (n 65) & $P$ value & Odds Ratio $(95 \% \mathrm{Cl})$ & 37 (21.4\%) & \\
\hline Sex & & & 0.071 & $1.788(0.950,3.365)$ \\
\hline Male & $73(67.6 \%)$ & $35(53.8 \%)$ & & \\
\hline Female & 35 (32.4\%) & $30(46.2 \%)$ & & \\
\hline Age & & & $<0.001$ & $10.038(4.749,21.218)$ \\
\hline$\leq 70$ years & 75 (69.4\%) & $12(18.5 \%)$ & & \\
\hline$\geq 71$ years & $33(30.6 \%)$ & $53(81.5 \%)$ & & \\
\hline \multicolumn{5}{|l|}{ SIMD (decile) } \\
\hline $1-3$ & $45(41.7 \%)$ & $28(43.1 \%)$ & 0.856 & $0.944(0.507,1.759)$ \\
\hline $4-6$ & $31(28.7 \%)$ & $26(40.0 \%)$ & 0.126 & $0.604(0.316,1.155)$ \\
\hline$\geq 7$ & $31(28.7 \%)$ & $11(16.9 \%)$ & 0.080 & $1.976(0.914,4.272)$ \\
\hline Address not recognised & $1(0.9 \%)$ & $0(0.0 \%)$ & 0.437 & $\operatorname{Inf}($ Nan, Inf) \\
\hline
\end{tabular}


Citation: Khan KS, El-Zanati H, Mahmud S (2020) Can Age Adjusted Charlson Comorbidity Index Predict Prognosis in Hospitalised COVID-19 patients?. Ann Public Health Reports 4(1):115-121

\begin{tabular}{|c|c|c|c|c|}
\hline Lymphopenia (x 10*g/L) & $61(56.5 \%)$ & 40 (61.5\%) & 0.513 & $0.811(0.433,1.520)$ \\
\hline \multicolumn{5}{|l|}{ Neutrophils (x 10*g/L) } \\
\hline Decreased $(<2.0)$ & $2(1.8 \%)$ & $4(6.1 \%)$ & 0.134 & $0.288(0.051,1.617)$ \\
\hline Normal (2.0 - 7.5) & $73(67.6 \%)$ & $38(58.5 \%)$ & 0.225 & $1.482(0.784,2.802)$ \\
\hline Increased ( > 7.5) & $33(30.6 \%)$ & $23(35.4 \%)$ & 0.511 & $0.803(0.418,1.543)$ \\
\hline \multicolumn{5}{|l|}{ Platelets (x 10* g/L) } \\
\hline Decreased $(<140)$ & $10(9.3 \%)$ & $15(23.1 \%)$ & 0.012 & $0.340(0.143,0.812)$ \\
\hline Normal (140 - 450) & $94(87.0 \%)$ & 49 (75.4\%) & 0.050 & $2.192(0.989,4.860)$ \\
\hline Increased (> 450) & $4(3.7 \%)$ & $1(1.5 \%)$ & 0.410 & $2.462(0.269,22.513)$ \\
\hline Egfr $\geq 59(\mathrm{ml} / \mathrm{mn} / 1.73 \mathrm{~m} 2)$ & 85 (78.7\%) & $29(44.6 \%)$ & $<0.001$ & $4.588(2.343,8.982)$ \\
\hline CRP (mg/L) & & & 0.002 & $0.335(0.168,0.668)$ \\
\hline$\leq 100$ & $57(52.8 \%)$ & $50(76.9 \%)$ & & \\
\hline$\geq 101$ & $51(47.2 \%)$ & $15(23.1 \%)$ & & \\
\hline First RT-PCR swab positive & $91(84.3 \%)$ & $61(93.8 \%)$ & 0.061 & $0.351(0.113,1.094)$ \\
\hline \multicolumn{5}{|l|}{ Admission category } \\
\hline Group 1 & $94(87.0 \%)$ & $37(56.9 \%)$ & $<0.001$ & $5.081(2.410,10.712)$ \\
\hline Group 2 & $6(5.6 \%)$ & $16(24.6 \%)$ & $<0.001$ & $0.180(0.066,0.489)$ \\
\hline Group 3 & $8(7.4 \%)$ & $12(18.5 \%)$ & 0.028 & $0.353(0.136,0.918)$ \\
\hline \multicolumn{5}{|l|}{ CXR (BSTI Classification) } \\
\hline CVCXO & $10(9.2 \%)$ & $18(27.7 \%)$ & 0.001 & $0.266(0.114,0.622)$ \\
\hline CVCX1 & $53(49.1 \%)$ & $14(21.5 \%)$ & $<0.001$ & $4.256(2.048,8.844)$ \\
\hline CVCX2 & $40(37.0 \%)$ & $25(38.5 \%)$ & 0.851 & $0.941(0.499,1.775)$ \\
\hline CVCX3 & $3(2.8 \%)$ & $7(10.8 \%)$ & 0.029 & $0.237(0.059,0.950)$ \\
\hline Not done & $2(1.9 \%)$ & $1(1.5 \%)$ & 0.878 & $1.208(0.107,13.586)$ \\
\hline
\end{tabular}

Note: Data in parentheses are $95 \%$ confidence intervals.

$\mathrm{ACCl}=$ Age adjusted Charlson Comorbidity Index; SIMD = Scottish Index of Multiple Deprivation; eGFR = estimated glomerular filtration rate; $\mathrm{CRP}=\mathrm{C}$-reactive protein; RT-PCR = Reverse transcriptase polymerase chain reaction; BSTI = British Society of Thoracic Imaging.

\section{Outcome}



Most Healthy

Least Healthy

Figure 2: Shows the number of patients who were intubated, 30-day all-cause mortality and overall poor outcome in different ACCl groups. 
Citation: Khan KS, El-Zanati H, Mahmud S (2020) Can Age Adjusted Charlson Comorbidity Index Predict Prognosis in Hospitalised COVID-19 patients?. Ann Public Health Reports 4(1):115-121

Table 3: Outcome.

\begin{tabular}{|l|l|l|l|l|}
\hline & Low ACCI (n 108) & High ACCI (n 65) & P value & Odds Ratio (95\% CI) \\
\hline Transferred to critical care & $46(42.6 \%)$ & $2(3.1 \%)$ & $<\mathbf{0 . 0 0 1}$ & $23.371(5.436,100.482)$ \\
\hline Intubated & $33(30.6 \%)$ & $1(1.5 \%)$ & $<\mathbf{0 . 0 0 1}$ & $28.160(3.746,211.686)$ \\
\hline 30-day all-cause mortality & $17(15.7 \%)$ & $15(23.1 \%)$ & 0.229 & $0.623(0.287,1.352)$ \\
\hline Intubated and deceased & $12(11.1 \%)$ & $0(0.0 \%)$ & $\mathbf{0 . 0 0 5}$ & Inf (Nan, Inf) \\
\hline Poor outcome & $38(35.2 \%)$ & $16(24.6 \%)$ & 0.146 & $1.662(0.835,3.311)$ \\
\hline Discharged & $84(78.7 \%)$ & $41(63.1 \%)$ & $\mathbf{0 . 0 3 6}$ & $2.049(1.040,4.036)$ \\
\hline Still in-patient & $7(6.5 \%)$ & $9(13.8 \%)$ & 0.105 & $0.431(0.152,1.220)$ \\
\hline
\end{tabular}

Note: Data in parentheses are $95 \%$ confidence intervals.

$\mathrm{ACCl}=$ Age adjusted Charlson Comorbidity Index.

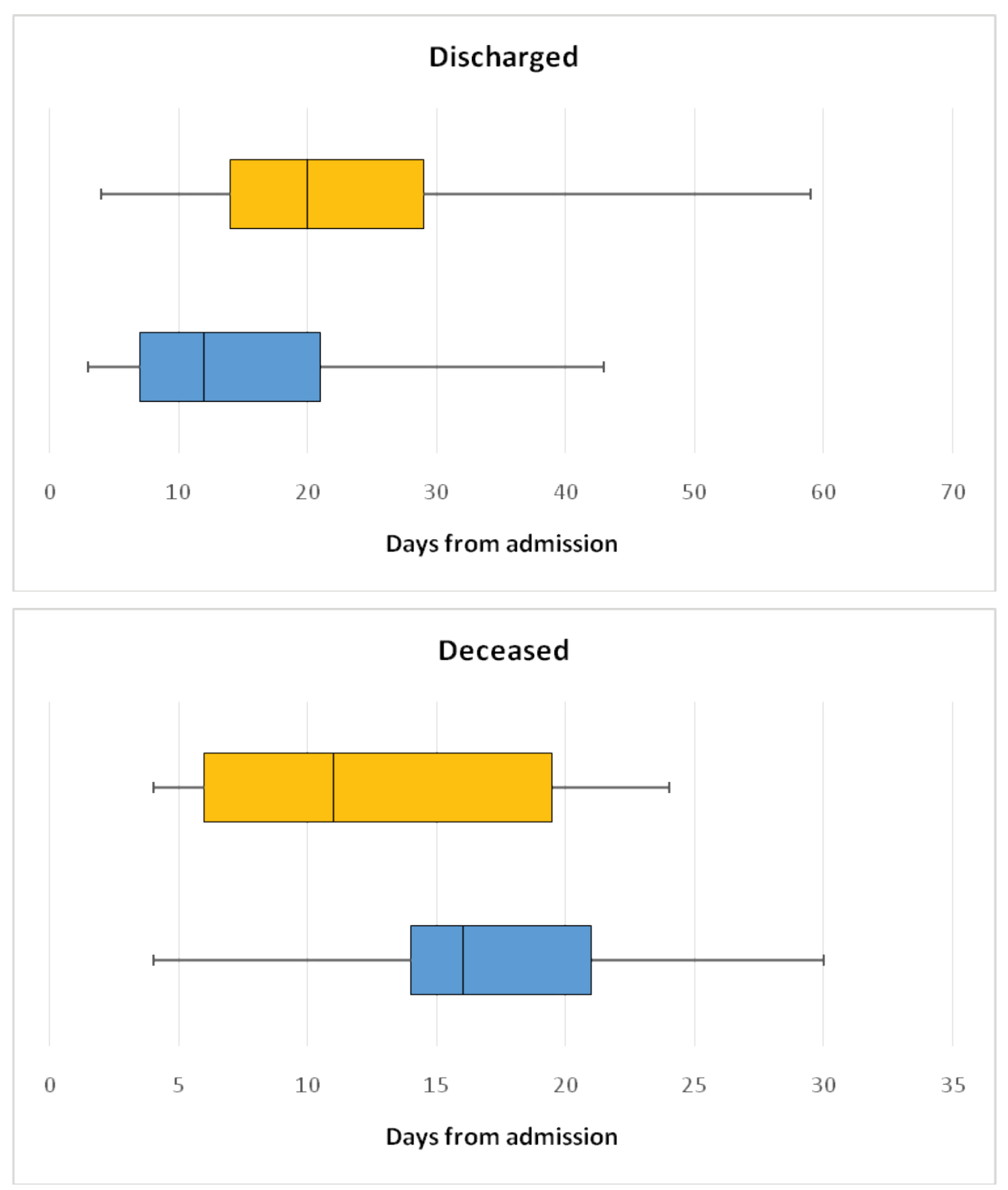

Figure 3: Total length of hospital stay in both groups for discharged and deceased patients. 
Citation: Khan KS, El-Zanati H, Mahmud S (2020) Can Age Adjusted Charlson Comorbidity Index Predict Prognosis in Hospitalised COVID-19 patients?. Ann Public Health Reports 4(1):115-121

There were more $\geq 71$-year's old patient in high $\mathrm{ACCl}$ (30.6\% vs. $81.5 \%, p<0.001$ ). High ACCl group were more likely to have low platelets $(9.3 \%$ vs. $23.1 \%$, p 0.012$)$. Low $\mathrm{ACCl}$ had more normal renal function $(78.7 \%$ vs. $44.6 \%, p<0.001)$. Low $\mathrm{ACCl}$ were more likely to present with the symptoms to COVID-19 (87.0\% vs. 56.9\%, p < 0.001) whereas high ACCI group were more likely to have COVI-19 diagnosis as an incidental finding $(5.6 \%$ vs. $24.6 \%, p<0.001)$ or contract it in hospital (7.4\% vs. 18.5\%, p 0.028). High ACCI were more likely to have index CXR normal (9.2\% vs. $27.7 \%, \mathrm{p} 0.001$ ) and low ACCI were more likely to have classical COVID-19 findings on index CXR (49.1\% vs. 21.5\%, p < 0.001) (Table 2).

Figure 2 and Table 3 compares the outcome of patients. More patients in low $\mathrm{ACCl}$ were transferred to critical care $(42.6 \%$ vs. $3.1 \%, p<0.001)$ and intubated $(30.6 \%$ vs. $1.5 \%, p$ $<0.001)$. However, the 30 -day all-cause mortality and overall poor outcome was similar in both groups, $(15.7 \%$ vs. $23.1 \%$, p 0.229 and $35.2 \%$ vs. $24.6 \%$, p 0.146 , respectively).

Figure 3 shows the LOS in hospital in both groups for patients who were discharged and who died. The median time to discharge was 8 days longer in high ACCl group (12 vs. 20 days, $\mathrm{p}$ 0.0012). The median time to death was 5 days longer in the low ACCI group (16 vs. 11 days, p 0.016).

\section{Discussion}

The original $\mathrm{CCl}$ paper published in 1987 has divided its scores into four categories after demonstrating that this had a direct correlation and value in prediction of one year mortality with $8 \%$ for a 0 score, $26 \%$ for scores of $1-2,52 \%$ for $3-4$ and $85 \%$ for scores $>4$ [4]. A large French study published few years ago including $>6$ million patients admitted to the hospital for various reasons has also confirmed this relation looking at 1 year mortality with the hazard ratio for an ACCI score of 3-4 being 20.3 and that for more than 4 being 77.3 [18]. Accordingly we have used a score of 4 to divide our cohort of patients into those with a high score and a low score due to the sharp rise in one year mortality previously demonstrated at this point.

It might seem to be a matter of common sense that patients with more comorbidities will do worse given the current published literature to date as previously highlighted. Our study has suggested that there were more patients transferred to critical care and intubated in the low $\mathrm{ACCl}$ group. This however can be explained by the selection bias of the anaesthetist, as a fitter patient is more likely to qualify for a bed in critical care if needed.

We however were expecting 30-day all-cause mortality to be higher in the high $\mathrm{ACCl}$ group. Again our data suggested that this wasn't true and although mortality was higher in the high ACCl group the difference was not statistically significant (Table 3).This might simply be a type 2 error due to a relatively small sample size however it might be possible that the ACCI is simply not sensitive enough for predicting short term outcomes as Charlson suggested herself in her original paper and most studies on this looked at longer term outcomes mostly 1 year mortality although some did report its value for predicting 30 day mortality [9] or in hospital mortality [19] .Another study with a similar number of patients to ours however reported that it wasn't a good predictor of outcomes in patients with respiratory failure in general [20] and a large Brazilian study again concluded that it doesn't predict outcomes in patients with severe acute respiratory infections [21].

A possible explanation could be the wide range of comorbidities included in the calculation of the ACCI while the comorbidities which are currently postulated to correlate with an adverse outcome with COVID-19 are quite limited namely hypertension, cardiac disease, chronic obstructive pulmonary disease (COPD), cerebrovascular disease (CVD) and diabetes mellitus [1-3]. Hypertension is not part of ACCl score. Whether other comorbidities do have a similar effect remains an unstudied point that needs further investigation given the huge impact it could have on triaging patients and determining prognosis and deciding upon the ceiling of care for these patients.

Our findings could also suggests that patients with multiple comorbidities don't necessary do as bad as one would expect, at least in the short term and therefore possibly shouldn't be denied life saving measures such as invasive ventilation based merely on their comorbidities and that a more individualized approach would be more appropriate.

A high $\mathrm{ACCl}$ did however correlate with a statistically significant longer hospital LOS. This is in going with the paper published by Charlson in 2014 suggesting that her index could also be used to predict higher future costs of medical care. Our high ACCl group also had a statistically significant shorter time to death compared to the low ACCl. One should however not read too much into these results but larger studies and perhaps meta-analyses would be needed to confirm these findings.

Our study does have several limitations including the relatively small sample size as previously discussed. We also only studied hospitalised patients which could make the results less general sable to the disease in the population overall. For LOS analysis we excluded the patients which acquired the COVID-19 infection during their hospital stay. This was to avoid skewing the data. 30-day mortality was also all-cause mortality as it is impossible to be certain the cause of death during the pandemic given the wide range of path physiological abnormality COVID-19 infection can cause.

\section{Conclusion}

Our study suggests that the ACCI has a poor predictive value for outcomes in COVID-19 hospitalised patients. This would imply that we have to be more careful in decisions regarding allocating resources to these patients based on comorbidities particularly their ceiling of care. However, larger studies are required to confirm this and perhaps a meta-analysis of these results. Also testing the predictive value of other comorbidity indices with regards to the disease would be needed before any firm conclusions could be reached with regards the effect of comorbidities on outcomes in COVID-19 patients.

\section{Acknowledgement}

None. 


\section{Conflict of Interest}

The authors declare that they have no competing interests.

\section{References}

1. Grasselli G, Zangrillo A, Zanella A, et al. (2020) Baseline characteristics and outcomes of 1591 patients infected with SARSCoV-2 admitted to ICUs of the lombardy region, Italy. JAMA 323: 1574-1581.

2. Yang $X, Y u Y, X u$ J, et al. (2020) Clinical course and outcomes of critically ill patients with SARS-CoV-2 pneumonia in Wuhan, China: A single-centered, retrospective, observational study. Lancet Respir Med 8: 475-481.

3. Li B, Yang J, Zhao F, et al. (2020) Prevalence and impact of cardiovascular metabolic diseases on COVID-19 in China. Clin Res Cardiol 109: 531-538.

4. Charlson ME, Pompei P, Ales KL, et al. (1987) A new method of classifying prognostic comorbidity in longitudinal studies: Development and validation. J Chronic Dis 40: 373-383.

5. Newschaffer CJ, Bush TL, Penberthy LT (1997) Comorbidity measurement in elderly female breast cancer patients with administrative and medical records data. J Clin Epidemiol 50: 725-733.

6. Hall WH, Ramachandran R, Narayan S et al. (2004) An electronic application for rapidly calculating Charlson comorbidity score. BMC Cancer 4: 94.

7. Charlson M, Szatrowski TP, Peterson J, et al. (1994) Validation of a combined comorbidity index. J Clin Epidemiol 47: 1245-1251.

8. Crooks CJ, West J, Card TR (2015) A comparison of the recording of comorbidity in primary and secondary care by using the Charlson index to predict short-term and long-term survival in a routine linked data cohort. BMJ Open 5: e007974.

9. Marchena-Gomez J, Acosta-Merida MA, Hemmersbach-Miller $M$, et al. (2009) The age-adjusted Charlson comorbidity index as an outcome predictor of patients with acute mesenteric ischemia. Ann Vasc Surg 23: 458-464.
10. Núñez JE, Núñez E, Fácila L, et al. (2004) Papel del índice de Charlsonen el pronóstico a 30 días y 1 añotras un infartoagudo de miocardio [Prognostic value of Charlson comorbidity index at 30 days and 1 year after acute myocardial infarction]. Revista Española de Cardiología 57: 842-849.

11. Murray SB, Bates DW, Ngo L, et al. (2006) Charlson index is associated with one-year mortality in emergency department patients with suspected infection. Acad Emerg Med 13: 530-536.

12. Chuang MH, Chuang TL, Huang KY, et al. (2017) Age-adjusted Charlson comorbidity index scores predict major adverse cardiovascular events and all-cause mortality among systemic lupus erythematosus patients. Ci Ji Yi Xue Za Zhi 9: 154-158.

13. Goldstein LB, Samsa GP, Matchar DB, et al. (2004) Charlson index comorbidity adjustment for ischemic stroke outcome studies. Stroke 35: 1941-1945.

14. Hemmelgarn BR, Manns BJ, Quan H, et al. (2003) Adapting the Charlson comorbidity index for use in patients with ESRD. Am J Kidney Dis 42: 125-132.

15. Poses RM, McClish DK, Smith WR, et al. (1996) Prediction of survival of critically ill patients by admission comorbidity. J Clin Epidemiol 49: 743-747.

16. (2020) Scottish Index of Multiple Deprivation.

17. (2020) Updated BSTI COVID-19 guidance for the reporting radiologist. The British Society of Thoracic Imaging.

18. Bannay A, Chaignot $C$, Blotière $P O$, et al. (2016) The best use of the Charlson comorbidity index with electronic health care database to predict mortality. Med Care 54: 188-194.

19. Sundararajan V, Henderson T, Perry C, et al. (2004) New ICD-10 version of the Charlson comorbidity index predicted in-hospital mortality. J Clin Epidemiol 57: 1288-1294.

20. Meireles M, Machado A, Lopes J, et al. (2018) Age-adjusted Charlson comorbidity index does not predict outcomes in patients submitted to noninvasive ventilation. Arch Bronconeumol 54: 503-509.

21. Setter NW, Peres ML, de Almeida BMM, et al. (2020) Charlson comorbidity index scores and in-hospital prognosis with severe acute respiratory infections. Intern Med J 50: 691-697.

DOI: $10.36959 / 856 / 506$

Copyright: ( $\subsetneq 2020$ Khan KS, et al. This is an open-access article distributed under the terms of the Creative Commons Attribution License, which permits unrestricted use, distribution, and reproduction in any medium, provided the original author and source are credited. 